\title{
Spontaneously Resolved Hepatic Artery Aneurysm Complicating A
} Laparoscopic Cholecystectomy

Y. El Badri ${ }^{1 *}$, F. Adjimabou ${ }^{1}$, B.Boutakioute ${ }^{1}$, M. Ouali Idrissi ${ }^{1}$, N. Cherif Idrissi Ganouni ${ }^{1}$

${ }^{1}$ Radiology Department, Arrazi Hospital, Mohammed VI university hospital, Cadi Ayyad University, Marrakech

DOI: $10.36347 /$ sjmcr.2021.v09i03.029

| Received: 23.02.2021 | Accepted: 06.03.2021 | Published: 30.03.2021

*Corresponding author: Y. El Badri

\section{Abstract}

Vascular complications following a laparoscopic cholecystectomy are less common but more serious with higher morbidity than bile duct damages. We report the case of a 79-year-old female who presented 20 days after laparoscopic cholecystectomy hepatic colic, jaundice and hematemesis. The ultrasound - Doppler / and abdominal CT angiography showed a pseudoaneurysm of the right hepatic artery compressing the bile ducts causing a dilation upstream. The evolution was marked by the spontaneous resolution of the clinical signs with complete resolution of the pseudoaneurysme in the angiography performed 48 hours later.

Keywords: cholecystectomy, pseudo aneurysm, hepatic artery, CT angiography.

Copyright $(\mathcal{C} 2021$ The Author(s): This is an open-access article distributed under the terms of the Creative Commons Attribution 4.0 International License (CC BY-NC 4.0) which permits unrestricted use, distribution, and reproduction in any medium for non-commercial use provided the original author and source are credited.

\section{INTRODUCTION}

Vascular complications following a laparoscopic cholecystectomy are less common but more serious with higher morbidity than bile duct damages [1].

Among these complications is the hepatic artery pseudoaneurysm. It is reported to occur in $0.6 \%$ of procedures. It was first described by Sandblom in 1948 as an unusual complication of trauma, of which percutaneous and laparoscopic maneuvers system represent the majority of the causes [2].

\section{CASE REPORT}

We report the case of a 79-year-old woman admitted to the emergency room for pain in the right hypochondrium and vomiting, associated with mucocutaneous jaundice, occurring 20 days after laparoscopic cholecystectomy for sclerotrophic gallbladder with lithiasis of the main bile duct. In the emergency room, the blood pressure was $10 / 6 \mathrm{mmHg}$ and the pulse was $80 \mathrm{bpm}$. The abdominal exam was normal. Hemoglobin rate was $9.4 \mathrm{~g} / \mathrm{L}$ and liver function tests were disturbed $(\mathrm{AST}=98 \mathrm{IU} / \mathrm{L}, \mathrm{ALAT}$ $=87 \mathrm{IU} / \mathrm{L}$, total bilirubin $77 \mathrm{mg} / \mathrm{L}$, direct bilirubin $75.4 \mathrm{mg} / \mathrm{L}$ ). Abdominal Doppler ultrasound revealed an intra hepatic cystic mass with a discontinuous regular wall, with arterial blood flow on color Doppler, in continuity with the right hepatic artery suggesting a pseudoaneurysm (Fig1). The abdominal CT angiography confirmed the diagnosis showing an intrahepatic pseudoaneurysm of the right hepatic artery measuring $23 \times 19 \mathrm{~mm}$, compressing the bile ducts responsible for the dilation of the upstream bile ducts (Fig 2). 

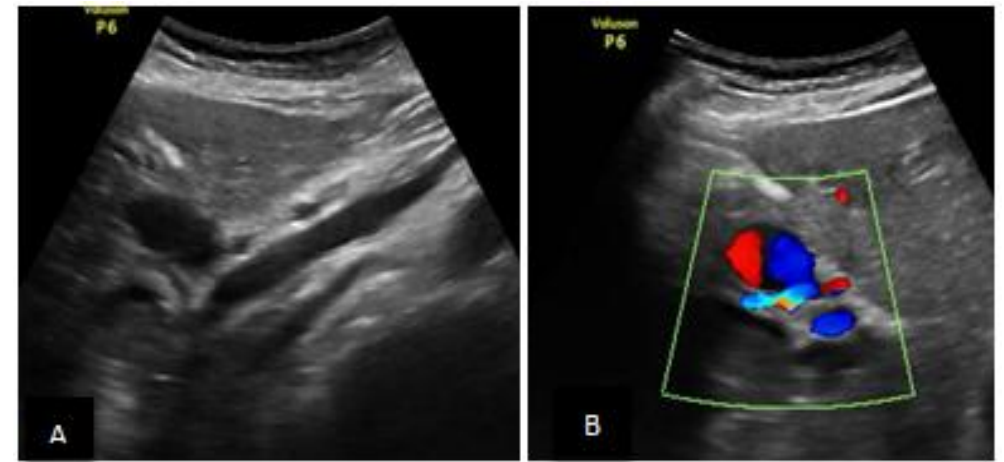

Fig-1: Longitudinal and transverse ultrasound images passing through the hepatic hilum and the left liver; in 2D mode (A) and in Doppler color (B) showing aneurysmal dilation of the right branch of the hepatic artery
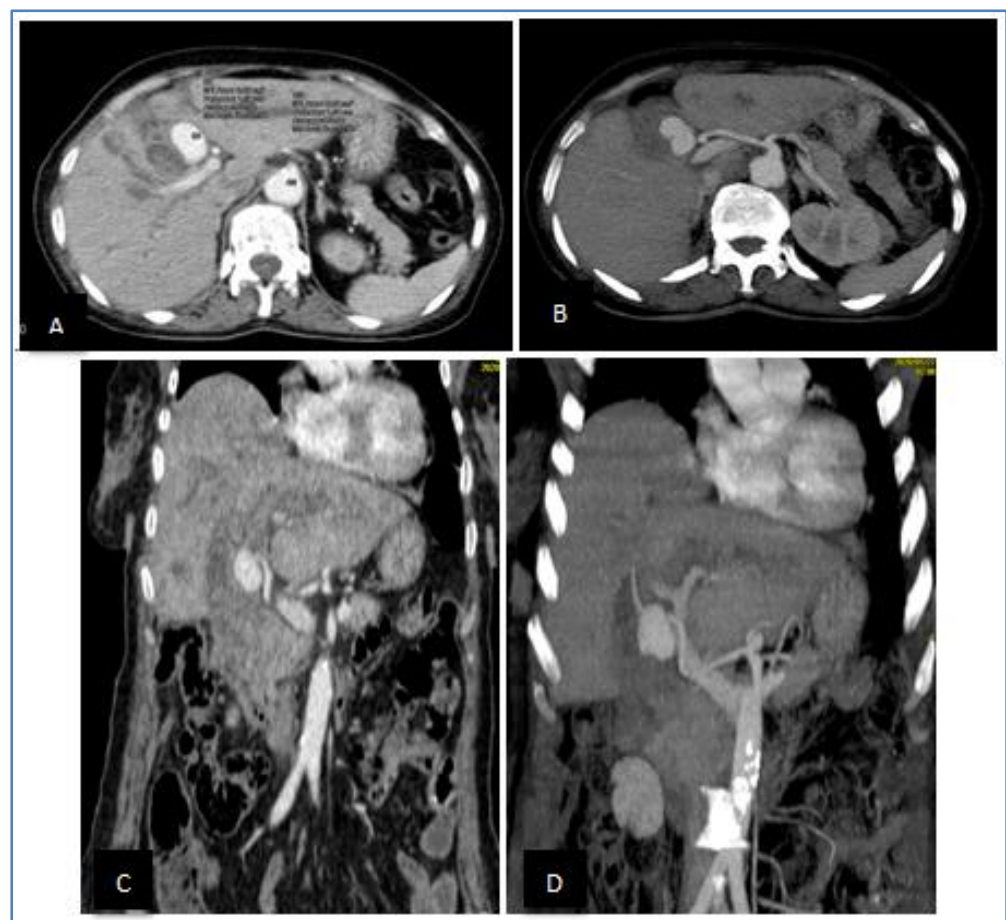

Fig-2: Arterial CT showing the pseudoaneurysm of the right branch of the hepatic artery compressing the nearby bile ducts causing their dilation. -In axial sections without (A) and with MIP reconstruction (B) - In coronal sections without (C) and with MIP reconstruction (D)

Two days after her admission, the patient became clinically asymptomatic with resolution of the jaundice. Embolization treatment was considered but pre-embolization arteriography did not reveal any abnormalities (Fig 3). A control abdominal CT angiography was therefore performed showing the complete and spontaneous resolution of the pseudoaneurysm (spontaneously thrombosed) (Fig 4).

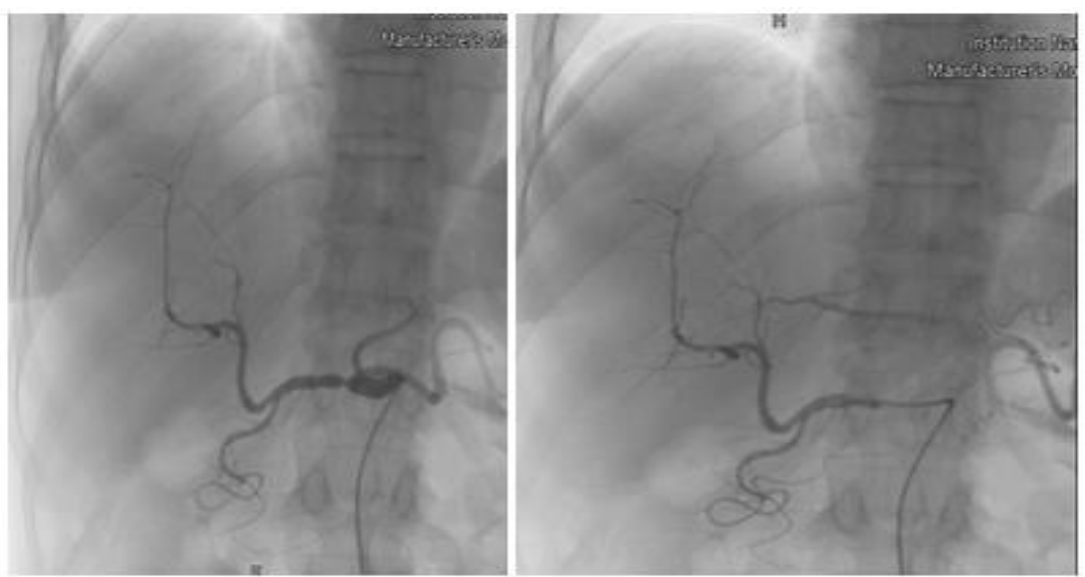

Fig-3: Arteriography showing good opacification of the celiac trunk and its dividing branches without visible aneurysmal dilation 

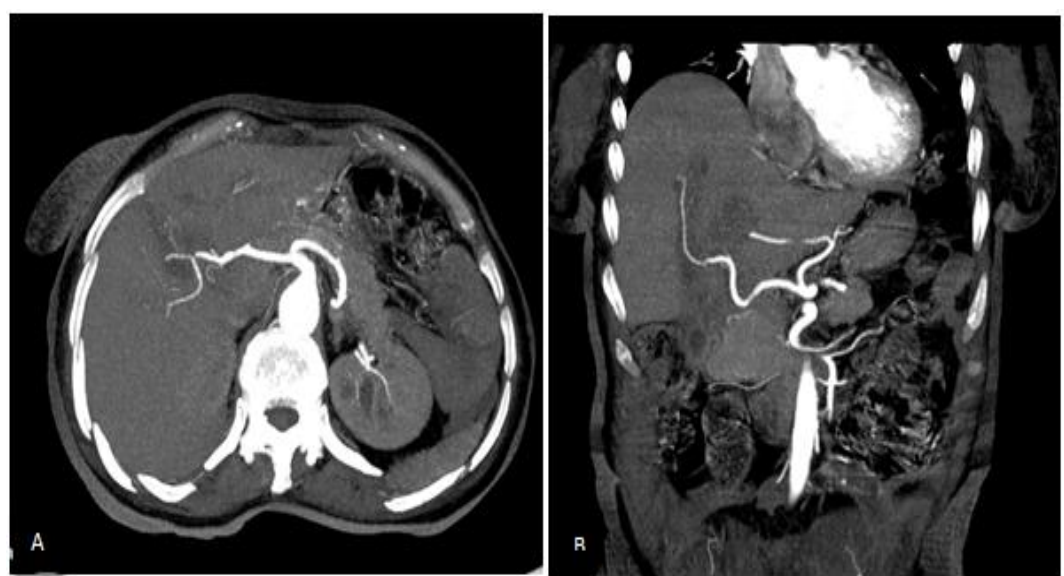

Fig-4: Control CT angiography after contrast injection in arterial phase, A- in axial section with MIP reconstruction, B- in coronal reformation with MIP reconstruction, Hypodense mass at the site of the pseudoaneurysm corresponding to its thrombosis with a normal right hepatic artery (normal caliber, regular and well opacified)

\section{DiscUSSION}

The pseudoaneurysms of the hepatic artery represent only $20 \%$ of aneurysms of the digestive arteries. It is the second most common location after the splenic artery and only $17-25 \%$ of them are intrahepatic. Their risk of rupture is higher than for other digestive sites [3].

This incidence is linked to the development of interventional radiological procedures on the liver and to the improvement of diagnostic imaging techniques. In fact, hepatic pseudoaneurysms occur either following an interventional radiology procedure, or after hepatic trauma or hepatobiliary surgery [4].

Hepatic pseudoaneurysm is a rare complication of laparoscopic cholecystectomy. Its incidence have been estimated to $0.6 \%$ over a series of 1513 procedures [5]. It can occur early, but if it occurs late, thermal injury (electrocoagulation) is the most likely cause $[6,7]$.

The natural evolution of the hepatic aneurysms is a gradual increase in their size, which can lead to rupture requiring an appropriate and rapid management. This complication is dangerous and occurs in 30 to $40 \%$ of cases [8]. It can occur in the free peritoneum or in adjacent organs: bile ducts with possible Quincke's triad (hepatic colic, jaundice and hemobilia), gastrointestinal tract, portal vein or Wirsung duct $[4,8]$.The death rate is around $20 \%$ [4].

The aneurysmal thrombosis, often asymptomatic, can constitute a mode of cure. Our patient was symptomatic at the same time, having presented the Quincke's triad without, however, evidence of communication with the bile ducts on imaging; then spontaneous clinical and radiological resolution making our case very unique.

In the absence of complications, the aneurysm remains silent and is discovered incidentally during imaging. Computed tomography remains the gold standard, performed in helical mode before and after intravenous injection of contrast. The arterial phase identifies, with great anatomical precision, the lesion as an intense and early enhancement similar to arterial enhancement. It also helps to diagnose a recent rupture in case of hyperdensity of the surrounding tissues, to detect perihepatic or retroperitoneal hematomas as well as hepatic perfusion disorders [4, 8]. It allows to identify the responsible artery and to analyze the coeliomaenteric tree [4]. However, the CT angiography technique must be rigorous, requiring thin sections in order to avoid false negatives. Maximum intensity projection (MIP) reconstructions identify an addition image, similar to that obtained by angiography. Other less constant signs are a contrast medium leak or a collection with a liquid-liquid level or debris, corresponding to blood clots.

Abdominal ultrasound reveals the presence of a cystic lesion with a regular wall, next to the vessels of the hepatic pedicle or in continuity with them. Color doppler confirms with great specificity the vascular nature of the sac and its circulating character. Allowing to guide a possible percutaneous embolization as well as to control the effectiveness of the treatment and the evolution of the aneurysm sac.

Magnetic resonance angiography gives a noninvasive high-resolution angiography and completes the vascular analysis with a visceral and biliopancreatic study [4]. But, the most sensitive additional examination remains the arteriography. It is essential whenever the diagnosis of pseudo-aneurysm is suspected. Although it provides rich anatomical information about the sac and any associated lesions, it still an invasive procedure and should be reserved for cases where embolization is being considered [8].

Indeed, the management methods have evolved with the advent of interventional radiology techniques. Surgery should only be considered in unstable patients or after failure of endovascular 
treatment [9]. Embolization has become the therapy of choice. After selective catheterization, the use of microcoils allows the exclusion of the false aneurysm and the preservation of the arterial branch. The success rate ranges from 81 to $96 \%$ [8]. Complications such as hepatic or vesicular necrosis are less frequent with distal microcatheterization. However, conventional endovascular treatment is not always possible, especially with difficult vascular setup, intimal dissection, or a history of ligation or embolization.

A direct transhepatic approach of the pseudoaneurysm with a fine needle, under ultrasound or fluoroscopic control, is possible and allows occlusion by injection of thrombin or coils [3, 4].

Few complications of direct embolization procedures have been described: an abscessed intrahepatic hematoma complicated by a biliary fistula or a recurrence of pseudoaneurysm by migration of a coil, justifying the need for a radiological monitoring by ultrasound or CT angiography [3].

\section{CONCLUSION}

The occurrence of a hepatic aneurysm after laparoscopic cholecystectomy is rare, but its management is crucial given the life threatening risk of complications. Imaging plays an essential role in both diagnosis and management using endovascular and transhepatic embolization techniques. However, spontaneous recovery, although rare, remains possible even in symptomatic patients as illustrated in our case.

\section{REFERENCES}

1. Finley DS, Hinojosa MW, Paya M, Imagawa DK. Hepaticarterynpseudoaneurysm: a report of seven cases and a review of the literature. SurgToday. 2005;35:543-7.

2. Slaba S, Abed O, Mallak N, Nassar J. Embolisation trans-hépatique d'un anévrisme de l'artère hépatique post-cholécystectomie laparoscopique. Journal des maladies vasculaires. $2010 \mathrm{Dec}$ 1;35(6):355-8.

3. Marcheix B, Dambrin C, Cron C, Sledzianowski JF, Aguirre J, Suc B, Cerene A, Rousseau H. Embolisation transhépatique percutanée d'un pseudoanévrisme post-traumatique de l'artère hépatique. InAnnales de chirurgie 2004 Dec 1 (Vol. 129, No. 10, pp. 603-606). Elsevier Masson.

4. Tétreau R, Monneuse O, Allaouchiche B, Pilleul F. Pseudo-anévrisme hépatique post-traumatique: traitement percutané à la colle vasculaire. Journal de Radiologie. 2007 Jun 1;88(7-8):975-7.

5. Chen CC, Chen BB, Wang HP. Upper gastrointestinal bleeding owing to right hepatic artery pseudoaneurysm after laparoscopic cholecystectomy. Gastroenterology. 2009 Nov 1;137(5):e5-6.

6. Yao CA, Arnell TD. Hepatic artery pseudoaneurysm following laparoscopic cholecystectomy. The American Journal of Surgery. 2010 Jan 1;199(1):e10-1.

7. Milburn JA, Hussey JK, Bachoo P, Gunn IG. Right hepatic artery pseudoaneurysm thirteen months following laparoscopic cholecystectomy. EJVES Extra. 2007 Jan 1;13(1):1-3.

8. Kassem TW. Right hepatic artery pseudoaneurysm as complication of laparoscopic cholecystectomy. The Egyptian Journal of Radiology and Nuclear Medicine. 2017 Dec 1;48(4):931-3.

9. Panda N, Narasimhan M, Gunaraj A, Ardhanari R. Laparoscopic management of postcholecystectomy sectoral artery pseudoaneurysm. Journal of minimal access surgery. 2014 Jan;10(1):37. 